Digital Press Social Sciences and Humanities

Analyse de la réflexion des phonèmes des mots français empruntés en indonésien

Andi Wete Polili and Pina Adelina Manullang

Proceeding of Conférence internationale sur le français 2018

Joesana Tjahjani, Merry Andriani, Sajarwa, Wening Udasmoro (eds) 


\title{
Analyse de la réflexion des phonèmes des mots français empruntés en indonésien
}

\author{
Andi Wete Polili* et Pina Adelina Manullang \\ Program Studi Bahasa Prancis Fakultas Bahasa dan Seni Universitas Negeri Medan, Sumatera Utara, Indonesia \\ *e-mail : wetepolili_andi@yahoo.com
}

\section{Résumé}

La recherche a pour but de savoir la réflexion des phonèmes et le type des changements du son des mots français empruntés en indonésien qui s'oriente du livre : Kamus Kata-Kata Serapan Asing dalam Bahasa Indonesia. Dans cette recherche, la méthode utilisée est la méthode descriptive qualitative. Selon le résultat de la recherche, on trouve qu'il y a des mots français empruntés. Il y a des changements, ce sont : la paragoge reflétant le son $[\varnothing] \rightarrow[\mathrm{i}],[\mathrm{e}],[\mathrm{t}],[\mathrm{l}],[\mathrm{r}]$, l'apocope reflétant $[\tilde{\jmath}] \rightarrow[\varnothing]$, l'épenthèse reflétant $[\varnothing] \rightarrow$ $[\mathrm{e}]$, la prothésiste reflétant $[\varnothing] \rightarrow[\mathrm{h}]$, la syncope reflétant $[\mathrm{w}] \rightarrow[\varnothing]$

\section{Mots-clés}

analyse, réflexion, changement, son, mot emprunté

\begin{abstract}
The purpose of the research is to find out the reflection of phonemes and the type of sound changes of the French words borrowed in Indonesian that orients itself from the book: Kamus Kata-Kata Serapan Asing Dalam Bahasa Indonesia. In this research, the method used is the qualitative descriptive method. According to the research, there are borrowed French words, and there are changes, such as the paragraph reflecting the sound [Ø] [i],[e],[t], [l], [r], the apocope reflecting [ग] [Ø], the spelling reflecting $[\varnothing][\mathrm{e}]$, reflective prosthesist [Ø] [h], reflective syncope [w] [Ø].
\end{abstract}

\section{Keywords}

analysis, reflection, change, sound, borrowed word

\section{Introduction}

\subsection{Arrière-plan}

L'utilisation des mots empruntés dans une langue ajoute l'enrichissement du vocabulaire en langue particulière. L'indonésien emprunte quelques mots français. Des mots français empruntés en indonésien se produisent en lexicale, du son, et des autres. Les exemples des mots français qui ont emprunté en indonésien comme ci-dessous:

- $\quad$ adoption [adopsjõ]

$\rightarrow$ adopsi [adopsi]

- $\quad$ barricade [baRikad] $\rightarrow$ barikade [barikade]

- alarme [alaRm] $\rightarrow$ alarm [alarm]

La source : Kamus kata-kata serapan asing dalam Bahasa Indonesia de J.S. Badudu en 2009.

Dans le processus de l'emprunte se produit le changement du son qui a entraîné le son des mots de l'indonésien et le français étant différents, on peut voir les exemples des changements le son des mots précédents:

1. adoption [adəpsj̃̃] $\rightarrow$ adopsi[adopsi]

Le mot adoption [adəpsjõ] en français a changé après avoir été emprunté en indonésien devenant adopsi [adopsi]. Le changement du son qui se produit est la disparation du son [ग̃] à la fin du mot (l'apocope). Cette disparation du son [õ] se fait à cause de l'abrègement du mot. 
Langue étrangère, le français qui a été emprunté en indonésien, on ne sait pas souvent que ces mots que nous utilisons dans nos activités quotidiennes, comme alarm de mot alarme, aksesori de mot accessoire, apartemen de mot appartement, est dérivé de la langue française.

Le système phonologique indonésien est différent celui du français. Lorsque l'indonésien emprunte des mots du français, l'écrit et la prononciation des empruntes de mots ont ajusté à la règle indonésienne, par conséquent, des mots français qui ont emprunté en indonésien sont changés. L'étude des emprunts entretient des relations avec plusieurs disciplines linguistiques comme la lexicologie, la morphologie, la syntaxe, la sémantique, et la phonologie (Haff :15). En se fondant sur les choses problématiques, quelle est la réflexion des phonèmes et le type des changements du son des mots français empruntés en indonésien?

\subsection{Changement du son}

Selon Crowley (2010), il existe trois types des changements du son, c'est-à-dire, (a) le changement phonétique sans changer phonèmes, (b) la modification du changement phonétique de phonèmes, et (c) les changements dans les phonèmes sans changement phonétique. Des changements le son qui sera abordé dans cette étude est des changements le son qui ne provoque pas le changement de sens. Crowley dit qu'il se trouve des catégories de changement du son en langues, ce sont :

1. La lenition

Le premier type de changement est la lenition. Le concept de la lenition et de la fortition ne sont pas très bien défini, et les linguistes qui utilisent le terme semblent souvent s'appuyer davantage sur l'intuition ou les conjectures que sur une compréhension détaillée de ce qu'est vraiment la lenition et la fortition. Les linguistes parlent parfois de certains sons comme relativement plus forts ou plus faibles que d'autres. De même, les sons peuvent sonores être considérés comme «plus forts» que les sons sourde, et les occlusives plus forts que les constituants; les consonnes plus forts que les semivoyelles; Les oraux sont plus forts que les sons glottiques; Et les voyelles avant et arrière sont plus forts que les voyelles centrales. La lenition et la fortition répartis sur :

a. L'aphérèse : Disparation le son à l'initial du mot. En général, la disparation de la consonne ou voyelle à initiale du mot.

Exemples des mots de langue angkamuthi à l'Australie :

- maji [maji] $\rightarrow$ aji [aji] (les aliments), disparation le son [m] à l'initiale du mot.

- wapun [wapun] $\rightarrow$ apun [apun] (la tête), disparation le son [w] à l'initiale du mot.

b. L'apocope : Disparation le son à la fin du mot ce qui est un changement son qui se produi très fréquent dans plusieurs langues.

Exemple du mot de français :

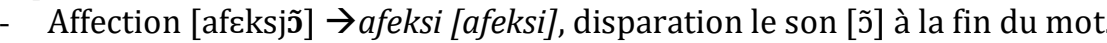

c. La syncope : Disparation le son à centre du mot.

Exemple du mot d'Anglais:

- Consumer ['kan'sjumar] $\rightarrow$ konsumen [konsumen], en l'anglais le son [j] à centre du mot est disparu.

d. Le cluster réduction : Disparation d'une consonne ou son de deux consonnes. Groupe consonantique est des consonnes consécutives en un mot sans voyelle est inséré entre eux.

Exemple le mot français:

ballon [ballis̃] $\rightarrow$ balon [balon], le son [1] en français est disparu de deux consonnes en indonésien.

2. L'addition du son, répartis sur :

a. L'excrescence : Addition le son consonne à centre deux consonnes dans un mot. Exemple:

L'excrescence a eu lieu en d'autres termes dans l'histoire d'anglais, et la consonne ajoutée et maintenant même représentée dans le système d'orthographe:

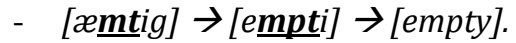

b. L'épenthèse : L'addition voyelle entre deux consonnes en mot, en particulier les mots d'empruntes pour ajuster le modèle des emprunteurs phonologiques. L'addition de ces sons souvent appelé anaptyxis.

Exemple le mot indonésien:

- putra [putra] $\rightarrow$ putera [putara], l'addition du son voyelle [ə] entre deux consonnes pour faciliter de la prononciation.

c. La prothésiste : Addition le son au initial du mot.

Exemple de langue en Papua New Guinea :

- api [api] $\rightarrow$ lahi [lahi], quand un mot a commencé par le son [a], une prothétique [1] a été ajoutée avant ça. 
d. La paragoge : Addition le son à la fin du mot.

Exemple de français:

camuflage [ka.mu.flaz] $\rightarrow$ kamuflase [kamuflase],l'addition du son [e] à la fin du mot kamuflase [kamuflase] en indonésien.

\subsection{Emprunte}

Le contact entre des langues fait que dans chaque langue une frange du lexique est d'origine étrangère (Paillard 2000:105). Guilbert (1975:89) dit que la collecte de mots, les mots d'empruntés, emprunter des mots devient être un phénomène linguistique dont l'étude est parallèle à l'histoire de la formation d'une langue.

Kridalaksana (2008:8) berkata bahwa "pinjaman" yaitu bunyi, fonem, unsur gramatikal atau unsur leksikal yang diambil dari bahasa lain. Kata serapan adalah kata yang diserap dari berbagai bahasa lain, baik dari bahasa daerah maupun dari bahasa asing, yang digunakan dalam bahasa Indonesia yang cara penulisannya mengalami perubahan ataupun tidak mengalami perubahan. C'est-à-dire, il dit que «emprunt» est le son, les phonèmes, les éléments grammaticaux ou les lexicaux provenant d'autres langues. Les mots empruntés qui sont emprunté d'autres langues, aussi bien en des langues locales et des langues étrangères, qu'utiliser en l'Indonésien que signifie ses écrits ont modifié ou n'ont pas modifié.

\subsection{Système phonologique français.}

Les voyelles françaises sur lieu d'articulation peuvent diviser en voyelles antérieures, et postérieures, et en mode d'articulation, les voyelles françaises peuvent être distinguées en fermées, mi-fermées, miouvertes/mi-basses, et ouvertes/basses.

Tableu 1 Le système d'articulation des voyelles françaises (Yuliati, 2015)

\begin{tabular}{|l|c|c|c|c|}
\hline \multirow{2}{*}{ Lieu d'articulation } & \multicolumn{2}{|c|}{ Antérieures } & \multicolumn{2}{c|}{ Postérieures } \\
\cline { 2 - 5 } Mode Articulatoire & Écartées & Arrondies & Écartées & Arrondies \\
\hline Fermées / hautes & & & & \\
\hline Mi- fermées / mi-hautes & $\mathrm{I}$ & $\mathrm{Y}$ & & $\mathrm{U}$ \\
& & $\emptyset$ & & 0 \\
\hline Mi-ouvertes / mi-basses & $\mathrm{E}$ & $\mathrm{OE}$ & & Õ \\
& $\tilde{\varepsilon}$ & $\tilde{\mathrm{CE}}$ & & \\
\hline Ouvertes / basses & $\mathrm{A}$ & & $\mathrm{a}$ & \\
& & & $\tilde{a}$ & \\
\hline
\end{tabular}

Sur le lieu d'articulation des consonnes françaises peut être divisée en consonnes bilabiale, labiodentale, apico-dentale, apico-alvéolaire, prédorso alvéolaire, prédorso palatale, dorso-palatale, et uvulaire, et en mode d'articulation, les consonnes françaises peuvent être distingués en occlusive, nasale, fricative, latérale, vibrante, glissante. 18 consonnes françaises peuvent être classifiées sur le lieu d'articulation et la mode d'articulation dans le tableau ci-dessous :

Tableu 2 Le système d'articulation des consonnes françaises (Yuliati, 2015)

\begin{tabular}{|l|c|c|l|l|l|l|l|l|l|l|}
\hline \multicolumn{2}{|l|}{$\begin{array}{l}\text { Mode } \\
\text { Arculatoire }\end{array}$} & $\begin{array}{l}\text { Lieu } \\
\text { d'articulation } \\
\text { biale }\end{array}$ & $\begin{array}{l}\text { Labio } \\
\text { Dentale }\end{array}$ & $\begin{array}{l}\text { Apico } \\
\text { Dentale }\end{array}$ & $\begin{array}{l}\text { Apico- } \\
\text { Alvéolaire }\end{array}$ & $\begin{array}{l}\text { Prédoro } \\
\text { Alvéolaire }\end{array}$ & $\begin{array}{l}\text { Prédorso } \\
\text { Palatale }\end{array}$ & $\begin{array}{l}\text { Dorso- } \\
\text { Palatale }\end{array}$ & $\begin{array}{l}\text { Dors- } \\
\text { Vélaire }\end{array}$ & Uvulaire \\
\hline & Sourde & $\mathrm{p}$ & & $\mathrm{t}$ & & & & & $\mathrm{k}$ & \\
\hline Nasale & Sonore & $\mathrm{b}$ & & $\mathrm{d}$ & & & & & $\mathrm{g}$ & \\
\hline
\end{tabular}




\begin{tabular}{|c|c|c|c|c|c|c|c|c|}
\hline \multirow[t]{2}{*}{ Fricative } & Sourde & $\mathrm{F}$ & & & & & & \\
\hline & Sonore & $\mathrm{v}$ & & $S$ & $\int$ & & & \\
\hline \multirow[t]{2}{*}{ Latérale } & Sourde & & & $\mathrm{Z}$ & $\mathrm{z}$ & & & \\
\hline & Sonore & & 1 & & & & & \\
\hline Vibrante & & & & & & & & $\mathrm{R}$ \\
\hline $\begin{array}{c}\text { Glissante } \\
\text { / Semi } \\
\text { Voyelle }\end{array}$ & & & & & & $4, j$ & w & \\
\hline
\end{tabular}

\subsection{Système phonologique indonésien}

Les voyelles indonésiennes sur le lieu d'articulation peuvent être divisée en voyelles antérieures, centre, et postérieure, et en mode d'articulation, les voyelles d'Indonésien peuvent être distinguées en hautes, mihautes, et basses.

Tableu 3 Le système d'articulation des voyelles indonésiennes (Muslich, 2010, p. 95)

\begin{tabular}{|c|c|c|c|}
\hline $\begin{array}{r}\text { Lieu } \\
\text { Mode } \\
\text { Arculatoire }\end{array}$ & Antérieures & Centre & Postérieures \\
\hline Hautes & $\mathrm{i}$ & & $\mathrm{u}$ \\
\hline Mi-hautes & $\mathrm{e}$ & ə & $\mathrm{o}$ \\
\hline Basses & & $\mathrm{a}$ & \\
\hline
\end{tabular}

Sur le lieu d'articulation des consonnes indonésiennes peuvent être distinguées en consonne bilabiale, labiodentale, apico-dentale, apico-alvéolaire, palato-alvéolaire, palatale, dorso-vélaire, et glottale, et sur la mode d'articulation, les consonnes indonésiennes peuvent être distinguées en occlusive, nasale, africative, fricative, latérale, vibrante, glissante. 21 consonnes indonésiennes peuvent être classifiées sur le lieu d'articulation et la mode d'articulation dans le tableau ci-dessous :

Tableu 4 Le système d'articulation des consonnes indonésiennes (Muslich, 2010, p. 95)

\begin{tabular}{|c|c|c|c|c|c|c|c|c|c|}
\hline \multicolumn{2}{|c|}{$\begin{array}{l}\text { Lieu } \\
\text { Mode } \\
\text { Arculatioire }\end{array}$} & Bila-biale & $\begin{array}{l}\text { Labio- } \\
\text { dentale }\end{array}$ & $\begin{array}{l}\text { Apico- } \\
\text { Dental }\end{array}$ & $\begin{array}{c}\text { Apico } \\
\text { Alvéolaire }\end{array}$ & $\begin{array}{l}\text { Palato } \\
\text { Alvéolaire }\end{array}$ & Palatale & $\begin{array}{l}\text { Dorso- } \\
\text { Vélaire }\end{array}$ & Glotale \\
\hline \multirow[t]{2}{*}{ Occlusive } & Sourde & $\mathrm{P}$ & & $\mathrm{t}$ & & & & $\mathrm{k}, \mathrm{q}$ & \\
\hline & Sonore & $\mathrm{b}$ & & $\mathrm{d}$ & & & & $\mathrm{g}$ & \\
\hline Nasale & Sourde & $\mathrm{m}$ & & $\mathrm{n}$ & & & & & \\
\hline Africative & & & & & & $c, j$ & & & \\
\hline \multirow[t]{2}{*}{ Fricative } & Sourde & & $\mathrm{f}$ & & $S$ & & & $\mathrm{x}$ & $\mathrm{h}$ \\
\hline & Sonore & & $\mathrm{v}$ & & $\mathrm{Z}$ & & & & \\
\hline \multirow{2}{*}{ Latérale } & Sourde & & & & & & & & \\
\hline & Sonore & & & & $\mathrm{L}$ & & & & \\
\hline Vibrante & & & & & $\mathrm{R}$ & & & & \\
\hline $\begin{array}{l}\text { Glissante } \\
\text { / Semi } \\
\text { Voyelle }\end{array}$ & & $\mathrm{w}$ & & & & & $\mathrm{y}$ & & \\
\hline
\end{tabular}




\section{Méthode de la recherche}

Denzin et Lincoln dans Emzir dans Joseph (2015, p. 38) expliquent la définition de la recherche qualitative; "Qualitative researche is multimethode in focus, involving an interpretive, naturalistic approach to its subject matter. This means that qualitative researchers study in their natural setting, attempting to make sense of or interpret phenomena in terms of the meanings people bring to them. Qualitative researche involves the studied use and collection of a variety of empirical materials - case study, personal experience, introspective, life story, interview, observational, historical, interactional, and visual text - that describe routine and problematic moment and meaning in individuals lives."

C'est-à-dire la recherche qualitative est multi méthode au point, impliquant une approche interprétative, naturaliste à son sujet. Cela signifie que les chercheurs qualitatifs étudient dans leur milieu naturel, en essayent de donner un sens ou interpréter les phénomènes en termes de sens que les gens apportent à eux. La recherche qualitative implique l'utilisation étudiée et collection d'une variété de matériaux empiriques, étude de cas, l'expérience personnelle, introspective, histoire de la vie, entretien, observation, historique, interaction, et des texte visuels qui décrivent la routine et le moment problématique et un sens dans la vie des individus.

Selon Setyadi (dans Lumbantobing 2012, p. 28), "data yang bersifat kualitatf yaitu yang digambarkan dengan kata-kata atau kalimat dan dapat dipisah-pisahkan menurut kategori untuk memperoleh kesimpulan." c'est-à-dire les données qualitatives sont des données qui sont décrites avec des mots ou des phrases et peuvent être séparées par catégorie pour obtenir une conclusion, c'est pourquoi on utilise la méthode qualitative.

\subsection{Source des données}

Les sources de recherche en utilisant les données issues de livres dans l'arrière-plan théorique et on utilise les résultats des recherches menées par des chercheurs précédents sous forme de données. Livres principaux que j'utilise sont les suivants : le livre Kamus Kata-Kata Serapan Asing dalam Bahasa Indonesia de J.S. Badudu en 2009, et le livre an introduction to historical linguistics de Terry Crowley en 2010 sera les sources de données.

\subsection{Technique de collecte des données}

Dans cette recherche, tous les changements du son des mots français empruntés en indonésien dans le livre kamus kata-kata serapan asing dalam bahasa Indonesia vont être analysés comme les données en suivant les étapes de cette recherche :

1. Trouver des mots français empruntés en indonésien.

2. Confirmer l'étymologie des mots emprunté à l'application dictionnaire français "Français" et dictionnaire indonésien " $K B B I V$ "

3. Trouver l'origine les mots empruntés en français.

4. Faire les transcriptions phonétiques de deux langues.

5. Trouver des mots français empruntés en indonésien qui change et ne change pas.

6. Identifier les types de changement du son selon la théorie de Crowley.

\section{Résultats et discussions}

Voilà le résultat de la recherche en forme du tableau ci-dessous :

Tableu 5 Tableau récapitulatif du résultat de la recherche

\begin{tabular}{|c|c|l|}
\hline No & Type de changement & \multicolumn{1}{c|}{ Les mots empruntés français en indonésien } \\
\hline 1. & Paragoge & 1. alliance [aljãs] $\rightarrow$ aliansi [aliansi] \\
& & 2.ambasade [ãbasad] $\rightarrow$ ambasade [ambasade] \\
& & 3. assonance [asonãs] $\rightarrow$ asonansi [asonansi] \\
& 4. attache [ataf] $\rightarrow$ atase [atase] \\
& 5. bagage [bagaz] $\rightarrow$ bagasi [bagasi]
\end{tabular}




\begin{tabular}{|c|c|c|}
\hline & & 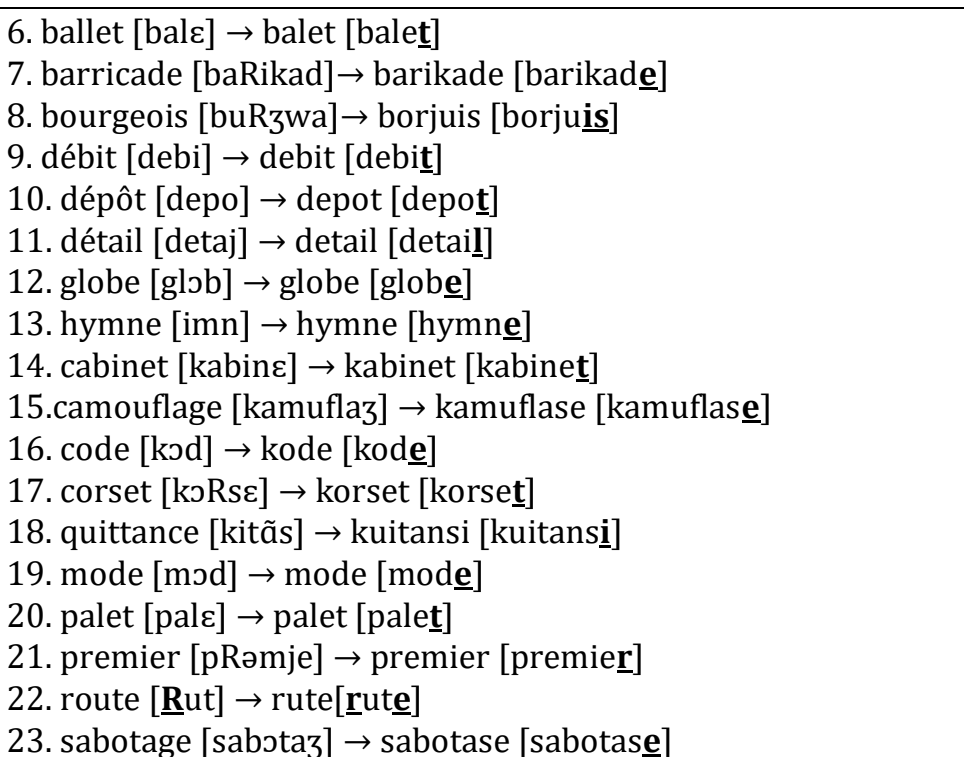 \\
\hline 2. & Apocope & 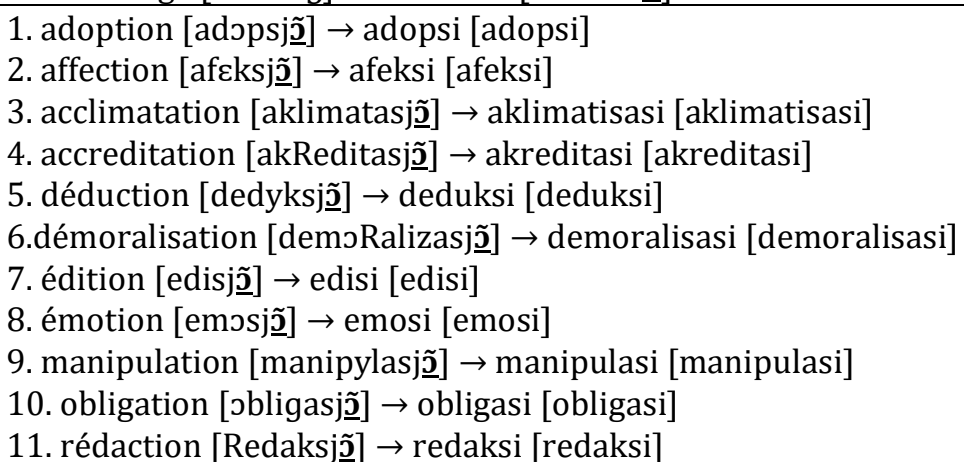 \\
\hline 3. & Epenthèse & 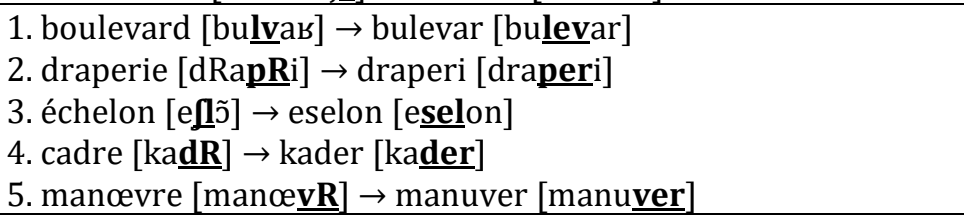 \\
\hline 4. & Prothésis & 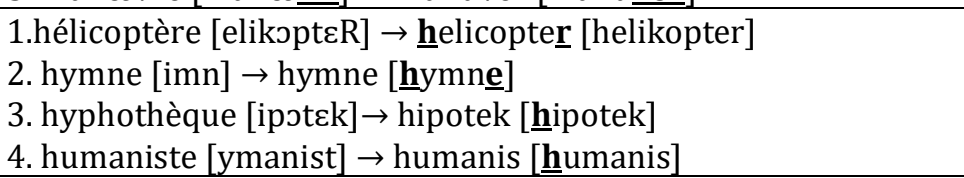 \\
\hline 5. & Syncope & 1. bivouac [bivwak] $\rightarrow$ bivak [bivak] \\
\hline
\end{tabular}

\subsection{Les changements du son des mots français empruntés en indonésien}

À partir des données qui ont été analysées, on obtient les changements du son, comme suivant:

1. La paragogue, c'est l'addition du son à la fin du mot.

$\begin{array}{ll}\text { C'est-à-dire : } & \bullet[\varnothing] \rightarrow[\mathrm{i}] \\ & \bullet[\varnothing] \rightarrow[\mathrm{e}] \\ & \bullet[\varnothing] \rightarrow[\mathrm{t}] \\ & \bullet[\varnothing] \rightarrow[\mathrm{l}] \\ & \bullet[\varnothing] \rightarrow[\mathrm{r}]\end{array}$

*le symbole [ø] signifie il n'existe pas.

2. L'apocope, c'est la disparation le sont à la fin du mot.

C'est-à-dire :

- [̃̃] $\rightarrow[\varnothing]$

3. L'épenthèse, c'est l'addition voyelle entre deux consonnes en mot pour séparer les deux consonnes.

C'est-à-dire :

$$
\begin{aligned}
& -[\mathrm{lv}] \rightarrow[\mathrm{lev}] \\
& -[\mathrm{dr}] \rightarrow[\mathrm{der}] \\
& \bullet[\mathrm{vr}] \rightarrow[\mathrm{ver}]
\end{aligned}
$$<smiles>CCCC(CC)[AlH2]Cl</smiles> 
4. La prothésiste, c'est l'addition du son à l'initial du mot.

C'est-à-dire : $\quad \bullet[\varnothing] \rightarrow[\mathrm{h}]$

5. La syncope, c'est la disparation le son à centre du mot.

C'est-à-dire : $\quad \bullet[\mathrm{w}] \rightarrow[\varnothing]$

\subsection{Analyse des données de la recherche}

Selon l'analyse de la recherche, on obtient enfin le résultat et l'analyse des données de la recherche. L'analyse de changement du son des mots français empruntés en indonésien, on trouve le type des changements, ce sont la paragoge, l'apocope, l'épenthèse, la prothésiste, la syncope, alors, c'est l'explication des changements des mots ci-dessous :

1. adoption [adopsj $\underline{\tilde{z}}$ ] $\rightarrow$ adopsi [adopsi]

Le changement du son qui se produit au mot français adoption [adopsj $\underline{\mathbf{s}}$ à adopsi [adopsi] en indonésien est le [õ] en français disparaît à la fin du mot en indonésien, c'est l’apocope.

2. acclimatation [aklimatasj $\underline{\mathbf{z}}$ ] $\rightarrow$ aklimatisasi [aklimatisasi]

Le changement du son qui se produit au mot français acclimatation [aklimatasjỗ] à aklimatisasi [aklimatisasi] en indonésien est le [õ] disparaît à la fin du mot, c'est l'apocope.

3. alliance [aljã $s] \rightarrow$ aliansi [aliansi $\underline{\underline{a}}]$

Le changement du son paragoge, le [i] est ajouté à la fin du mot indonésien.

4. ambasade [âabasad] $\rightarrow$ ambasade [ $\underline{\text { ambasade }]}$

Le changement du son la paragoge le [e] est ajouté a la fin du mot indonésien.

5. assonance [asonẫs] $\rightarrow$ asonansi [asonansi $\underline{1}]$

Le changement du son qui se produit au mot français assonance [asonãas] $\rightarrow$ asonansi [asonansi $\underline{\mathbf{i}}$ en indonésien est la paragoge, le son [i] est ajouté à la fin du mot indonésien.

6. bagage [bagaz] $\rightarrow$ bagasi [bagasi $\underline{i}$

Le changement du son qui se produit au mot français bagage [bagaz] à bagasi [bagasi] en indonésien est la paragoge parce que le [i] est ajouté à la fin du mot indonésien.

7. bivouac [bivwak] $\rightarrow$ bivak [bivak]

Le changement du son qui se produitau mot françaisbivouac [bivwak] àbivak [bivak] en indonésien est le [w] disparaît au centre du mot, c'est la syncope

8. boulevard [bulvaR] $\rightarrow$ bulevar [bulevar]

Le changement du son qui se produit au mot français boulevard [bulvaR] à bulevar [bulevar] en indonésien est l'épenthèse la voyelle [e] est ajouté entre les deux consonnes [l] et [v] du mot bulevar en indonésien pour séparer les deux consonnes, afin de se conformer au système phonologique de l'emprunteur.

9. débit [debi] $\rightarrow$ debit [debit $]$

Le changement du son qui se produit au mot français débit [debi] à debit [debit $]$ en indonésien est la paragoge parce que le [e] est ajouté à la fin du mot indonésien.

10.dépôt [depo] $\rightarrow \operatorname{depot}[\operatorname{depot}]$

Le changement du son qui se produit au mot français dépôt [depo] à depot [depot $]$ en indonésien est la paragoge parce que le [t] est ajouté à la fin du mot indonésien.

11. draperie [dㅁaㅁi] $\rightarrow$ draperi [draperi] Le changement l'épenthèse, la voyelle [e] est ajouté entre les deux consonnes [p] et [r] du mot draperi[draperi]en indonésien pour séparer les deux consonnes.

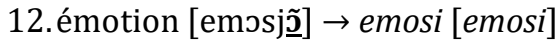

Le changement du son qui se produit au mot français émotion [emosj]̃̃ à emosi [emosi] en indonésien est le son [0̃] disparaît à la fin du mot en indonésien, c'est l'apocope.

13. globe [glob] $\rightarrow$ globe [globe $]$

Le changement du son qui se produit au mot français globe [glıb] à globe [globe] en indonésien est la paragoge, parce qu'il y a le[e] est ajouté à la fin du mot indonésien.

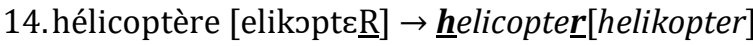

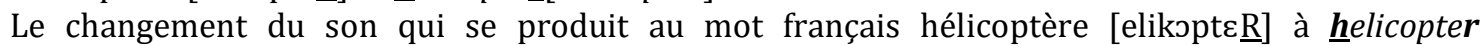
[helikopter] en indonésien sont la prosthésiste parce que le son [h] est ajouté à l'initial du mot indonésien.

15.hymne [imn] $\rightarrow$ hymne $[\underline{\text { himne }}]$

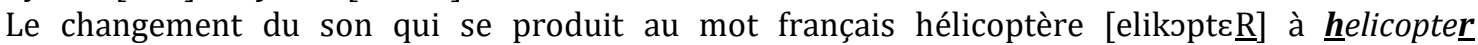
[helikopter] en indonésien est la prosthésiste parce que le [h] est ajouté à l'initial du mot indonésien,

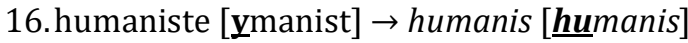


Le changement du son qui se produit au mot français humaniste [ymanist] $\rightarrow$ humanis [humanis] en indonésien est la prosthésiste parce que le son [h] est ajouté à l'initial du mot indonésien, ensuite, le $[y]$ français a remplacé par son $[u]$ ce qui est presque similaire en indonésien parce que le son $[y]$ n'existe pas en indonésien, le type des deux sons était la voyelle haute.

17. mode [mod] $\rightarrow$ mode [mode]

Le changement du son qui se produit au mot français mode [mod] à mode [mode] en indonésien est le changement du son la paragoge, le son [e] est ajouté à la fin du mot indonésien.

18. obligation [obligasj $\underline{\tilde{x}}$ ] $\rightarrow$ obligasi [obligasi]

Le changement du son qui se produit au mot français obligation [obligasj $\underline{\tilde{x}}$ ] à obligasi [obligasi] en indonésien est le son [ग̃] disparaît à la fin du mot en indonésien, c'est-à-dire l'apocope.

19.palet [pale] $\rightarrow$ palet $[$ palet $]$

Le changement du son qui se produit au mot français palet [pale] à palet [palet] en indonésien est le changement du son la paragoge, le [t] est ajouté à la fin du mot indonésien.

20.route $[\underline{\mathrm{Rut}}] \rightarrow$ rute $[\underline{\text { rute }}]$

Le changement du son qui se produit au mot français route [Rut] en français à rute [rute $]$ en indonésien est le changement du son la paragoge, le [e] est ajouté à la fin du mot indonésien.

\section{Conclusion}

Il se trouve des mots français empruntés en indonésien qui ont trouvé dans le livre kamus kata-kata serapan asing dalam bahasa indonesia. Il se trouve des mots qui ne changent pas, la prononciation en français est pareille qu'indonésien. Ensuite, il y a des mots qui changent en raison de la différencier au système phonologique de deux langues.

Les résultats de l'analyse des mots empruntés basés sur les données trouvées, on trouvé quelques tendances des changements du son qui se produisent lorsque le français a emprunté en l'indonésien, ces tendances sont :

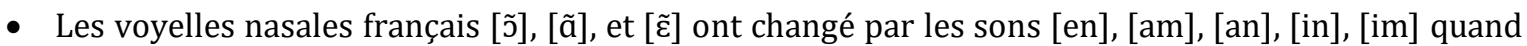
ils se prononcent en indonésien.

- Le [e] le son antérieure mi-haute voyelle française à la fin du mot comme les mots route [Rut], code [kod] et le son [h] à l'initial du mot comme hélicoptère [elikjpteR] ne sont pas prononcé en français mais en l'indonésien ils sont prononcés rute [rute], kode [kode] et helikopter [helikopter]. Les changements du son comme celui-ci se produisent parce que l'indonésien a tendance à adapter des mots français empruntés en ressemblant à l'orthographe française plutôt que la prononciation de ces mots.

\section{Références}

Akidah, M. A. (2013). Phonological and Semantic Change in Language Borrowing. International Journal of

Education and Research, 4, 3-9.

Badudu, J. (2009). Kamus Kata-Kata Serapan Asing dalam Bahasa Indonesia. Jakarta: Kompas.

Calvet, L.-J. (2007). La Sociolinguistique. Paris: Presses Universitaires de France.

Chaer, A. (2007). Linguistik Umum. Jakarta: Rineka Cipta.

Crowley, T. (2010). An Introduction to Historical Linguistics. Oxford: Oxford University Press.

Juliawan, M. D. (2017). An Analysis of Phonemic and Graphemic Changes of English Loanwords in Bahasa

Indonesia Appearing in Magazine “Chip." International Journal of Language and Literature, 1(1), 54-57.

Kridalaksana, H. (2007). Kamus Linguistik. Jakarta: Gramedia Pustaka Utama.

Loubier, C. (2011). De l'Usage de l'Emprunt Linguistique. Québec: Université de Québec.

Margono. (2011). Metode Peneletian Kualitatif. Semarang: Buana Jaya.

Muslich, M. (2010). Tinjauan Deskriptif Sistem Bunyi Bahasa Indonesia. Jakarta: Bumi Aksara. 
Peperkamp, S. (2005). A Psycholinguistic Theory of Loanword Adaptations. Paris: Université de Paris. https://doi.org/10.3765/bls.v30i1.919

Yuliati, R. (2015). Fonologi Bahasa Prancis. Puitika, 11(1), 26-35. Retrieved from http://jurnalpuitika.fib.unand.ac.id/index.php/jurnalpuitika/article/view/10 